\title{
Phenotypes of BPS/IC
}

\author{
Sachin Malde ${ }^{1} \cdot$ Arun Sahai $^{1}$
}

Published online: 17 July 2020

(C) The Author(s) 2020

\begin{abstract}
Purpose of Review The aim of this review is to summarise the latest research related to different phenotypes of BPS/IC, addressing the evidence for current well-defined phenotypes as well as identifying novel potential phenotypes and highlighting areas for future study.

Recent Findings Two distinct phenotypes of BPS/IC are well-recognised: Hunner's lesion disease and non-Hunner's lesion BPS/IC. Recent studies have shown these phenotypes exhibit distinct clinical, pathological and cystoscopic features, and targeted treatment to Hunner's lesions can prove effective. Recent studies have also identified new potential phenotypes based on biochemical, molecular and histological markers, pathophysiological mechanisms of disease, clinical features, cystoscopic findings, radiological features and urodynamic factors. This evidence has improved our understanding of the underlying mechanism of disease and may enable more personalised and targeted therapy in the future.

Summary Novel phenotypes of BPS/IC relate to the presence of certain biomarkers, alterations in the urinary microbiome, the characteristics of pain and presence of co-existing somatic and psychosocial conditions, altered patterns of brain white matter changes and urodynamic features. Further study is required to evaluate whether these potential phenotypes are clinically useful based on their ability to guide treatment selection and predict outcome from therapy, and therefore optimise therapeutic outcomes.
\end{abstract}

Keywords Bladder pain syndrome $\cdot$ Interstitial cystitis $\cdot$ Phenotype $\cdot$ Hunner's ulcer $\cdot$ Biomarker

\section{Introduction}

The symptom complex of persistent or recurrent pain, pressure or discomfort perceived in the urinary bladder region, accompanied by at least one other urinary symptom, such as pain worsening with bladder filling and daytime and/or nighttime urinary frequency or urgency, in the absence of other identifiable causes, is now widely defined as bladder pain syndrome/interstitial cystitis (BPS/IC) $[1 \bullet \bullet, 2 \bullet]$. Although exact definitions and nomenclature vary, these key symptomatic features have been adopted by all international guidelines in

This article is part of the Topical Collection on BPS/Interstitial Cystitis

Sachin Malde

Sachin.malde@gstt.nhs.uk

Arun Sahai

Arun.sahai@gstt.nhs.uk

1 Department of Urology, Guy's and St Thomas' NHS Foundation Trust, Great Maze Pond, London SE1 9RT, UK an attempt to standardise diagnosis, treatment and scientific research into this debilitating chronic condition [3]. However, it is recognised that the umbrella term BPS is likely to encompass numerous different underlying subgroups, or phenotypes, each with a different pathophysiological cause, unique clinical features and differing responses to therapy [4]. One such distinct clinical phenotype, the so-called Hunner lesion disease (HLD), has been recognised since the first descriptions of this condition over 100 years ago [5]. This is a clearly identifiable phenotype of BPS/IC with distinct clinical, cystoscopic and histopathological features, and for which specific lesion-directed therapies are available. Much of the evidence base on phenotyping is therefore related to the presence or absence of Hunner's lesions. The accurate phenotyping of patients with BPS/IC in this way is essential in order to offer personalised therapy and optimise treatment outcomes; the importance of phenotyping has also been recognised for optimising outcomes in other chronic bladder conditions [6].

Although HLD represents an accepted and widely studied phenotype of BPS/IC [7••], other less well-characterised methods to phenotype patients are based on the following: biochemical, 
molecular or histological markers, pathophysiological mechanisms of disease, clinical features, cystoscopic findings, radiological features and urodynamic factors (Table 1). Assessing response to different treatments may also facilitate our understanding of underlying mechanisms of disease and may thereby provide novel ways to phenotype patients.

The aim of this review is to discuss the latest evidence that may inform the way we phenotype patients with BPS and to highlight areas for future research.

\section{Biomarkers}

The diagnosis of BPS/IC is made clinically, based on the presence of the typical symptoms described above in the absence of any other identifiable cause. However, this symptombased diagnosis can lead to considerable variability and inaccuracy in diagnosis, as well as delay in treatment, and so an objective biomarker is desirable as a reproducible diagnostic and prognostic indicator of disease, its severity and in assessing response to treatment. Numerous biomarkers (from urine, blood, stool and bladder tissue) have been studied in recent years, and different phenotypes of BPS/IC may be identifiable by the presence or absence of certain biomarkers, or by assessing the effects of treatment on these markers.

\section{Urine Biomarkers}

Several urinary biomarkers have been evaluated as diagnostic and prognostic markers for BPS/IC, but their role in identifying different phenotypes of disease has not been widely studied [8].

\section{Phenotype Based on Elevated APF}

Early studies of antiproliferative factor (APF), a peptide that inhibits the proliferation of bladder epithelial cells in vitro, showed that it was significantly more abundant in the urine of patients with IC compared with control urine samples (sensitivity 94\%, specificity 95\%) [9]. This was confirmed in another study of 64 patients with BPS (38 with Hunner's lesions and 26 without), which found that $92 \%$ of IC patients had APF activity compared with only $3 \%$ of controls [10]. However, there was no statistically significant difference in APF levels between Hunner's lesion and non-Hunner's lesion patients, and although hydrodistension led to reduced APF levels, this

Table 1 Potential phenotypes of BPS/IC

\begin{tabular}{|c|c|}
\hline Feature & Phenotype \\
\hline Biomarkers (urine, serum, tissue) & $\begin{array}{l}\text { Presence of elevated levels of the following may } \\
\text { represent distinct phenotypes: } \\
\text { - APF } \\
\text { - MIF } \\
\text { - CXCL10 } \\
\text { - Etio-S } \\
\text { - TLR-4 } \\
\text { - WNT11 } \\
\text { - Angiogenesis markers }\end{array}$ \\
\hline Urinary microbiome & $\begin{array}{l}\text { 'Infection' phenotype (if nanobacteria, virus or fungus identified) } \\
\text { Phenotype based on altered lactobacillus levels }\end{array}$ \\
\hline Symptomatic & $\begin{array}{l}\text { Phenotype based on location of pain (bladder-centric vs. systemic) } \\
\text { Phenotype based on presence of functional somatic syndromes } \\
\text { Phenotype based on type of psychosocial comorbidity } \\
\text { (anxiety vs. depression vs. post-traumatic) }\end{array}$ \\
\hline Radiological & $\begin{array}{l}\text { Phenotype based on pelvic floor hypertonicity } \\
\text { Phenotype based on reduced bladder blood flow } \\
\text { Phenotype based on abnormal cerebral white matter changes }\end{array}$ \\
\hline Urodynamics & $\begin{array}{l}\text { Phenotype based on abnormal volume and sensation characteristics on } \\
\text { filling cystometry (volume-dependent vs. volume-independent pain) } \\
\text { Phenotype based on abnormal voiding cystometry }\end{array}$ \\
\hline Cystoscopic & $\begin{array}{l}\text { Phenotype based on presence of Hunner's lesions } \\
\text { Phenotype based on extent of Hunner's lesions } \\
\text { Phenotype based on low anaesthetic bladder capacity }\end{array}$ \\
\hline Histological & $\begin{array}{l}\text { Phenotype based on epithelial denudation and chronic inflammation } \\
\text { (lymphoplasmacytic and plasmacytic patterns) }\end{array}$ \\
\hline
\end{tabular}


did not correlate with symptomatic improvement [11]. Importantly, patients in both these studies had to meet the National Institute of Diabetes and Digestive and Kidney Diseases (NIDDK) diagnostic criteria for IC to be included, with evidence of either glomerulations or Hunner's lesions on cystoscopic assessment and a capacity of $<350 \mathrm{ml}$. These criteria are likely to represent a more severe spectrum of BPS/IC and are likely to exclude many patients that have BPS/IC but without any cystoscopic abnormalities. This suggests that APF may be a marker of a more severe phenotype of BPS/IC, but comparison against BPS/IC patients with normal cystoscopic findings and assessing response to different treatments, is still required to determine whether this biomarker can be used to identify a unique phenotype of BPS/IC.

\section{Phenotype Based on Elevated MIF or CXCL10}

The concentration of another urinary marker, macrophage migration inhibitory factor (MIF), was found to be approximately 5-fold higher in BPS/IC patients with Hunner's lesions compared with BPS/IC patients without Hunner's lesions and non-BPS/IC controls [12]. Elevated levels of this marker may therefore identify a specific phenotype of BPS/IC, but levels were also found to be increased in bacterial cystitis and radiation cystitis patients. Furthermore, response to treatment has not been studied and so it is unclear whether MIF can enable individualisation of therapy. Similarly, urinary CXCL10, a chemokine involved in recruiting proinflammatory cells, has been shown to discriminate between Hunner's and non-Hunner's lesion BP/IC with a sensitivity of $46.1 \%$ and specificity of $93.7 \%$, but its ability to predict response to treatment has also not been studied [13].

\section{Phenotype Based on Elevated Etio-S}

The most accurate urinary marker appears to be etiocholan$3 \alpha$-ol-17-one sulfate (Etio-S), a bioactive metabolite of testosterone. Although the mechanistic relationship between Etio-S and BPS/IC remains to be defined, elevated Etio-S was found to predict BPS/IC with a specificity of $87.4 \%$ and sensitivity of $91.2 \%$, and levels were found to discriminate between high and low symptom severity [14]. However, patients were not subdivided into those with or without Hunner's lesions and response to treatment has not been assessed.

\section{Serum Markers}

A recent Multidisciplinary Approach to the Study of Chronic Pelvic Pain (MAPP) study of 66 patients with BPS/IC found that increased Toll-like receptor 4 (TLR-4) inflammatory responses were associated with a greater likelihood of extrapelvic pain symptoms, suggesting that this could be a marker of a specific systemic phenotype of BPS/IC [15].

\section{Bladder Tissue Markers}

Numerous chemokines and inflammatory mediators have been studied as potential biomarkers for BPS/IC, but most require further validation to determine whether they can be used to identify specific subgroups of BPS/IC accurately [8, $16]$.

\section{Phenotype Based on Elevated WNT11}

Recently, a small study evaluated the WNT signalling cascade in bladder biopsies from 18 Hunner's lesion, 7 non-Hunner's lesion and 5 non-BPS/IC patients [17]. The WNT pathways are thought to have a complex role following tissue injury, promoting tissue regeneration and fibrosis. Overall WNT gene expression was found to be lower in non-Hunner's lesion patients, and specifically WNT11 was significantly downregulated in patients without Hunner's lesions. Potentially, the presence of this marker may identify patients that would benefit from specific anti-fibrotic therapy. Further study is required to assess whether WNT11 expression can be used to phenotype patients with BPS/IC in this way.

\section{Urinary Microbiome}

Recent technological advances have led to a greater understanding of the urinary microbiome and its potential role in the pathophysiology of several chronic bladder conditions. Whether BPS/IC is triggered by an infectious aetiology is a source of debate, but alterations in the urinary microbiome may represent another phenotype of BPS/IC that would respond to specific antimicrobial treatment. Although the absence of infection is a pre-requisite for the diagnosis of BPS/ IC, it is becoming clear that standard quantitative bacterial culture of a mid-stream urine specimen is less reliable than widely thought and many patients with BPS/IC may have an infectious cause that can be successfully treated with antimicrobial agents $[18,19]$. Using specific culture media with longer incubation periods led to the identification of a uropathogen in $50 \%$ of patients with BPS/IC, and symptomatic improvement was reported after appropriate antimicrobial treatment [20]. The use of $16 \mathrm{~S}$ ribosomal RNA nextgeneration sequencing (NGS) revealed the presence of nanobacteria in $41 \%$ of patients with BPS/IC (based on NIDDK criteria), and treatment with oral and intravesical tetracycline led to cure or improvement of symptoms in $91 \%$ of patients [21]. Others have found that the urinary microbiome in patients with IC is dominated by Lactobacillus species when compared with that of healthy asymptomatic females, and some investigators have detected a greater incidence of fungal isolates in patients with more severe symptoms [22, 23]. Greater levels of viral isolates (Epstein-Barr virus and Polyomavirus BK) have also been reported in patients with 
Hunner's lesions, with significant improvement in viral load and symptoms following intravesical anti-viral treatment [24-26]. Studying alterations in the urinary microbiome is a promising area for further research and may enable more accurate phenotyping and targeting of therapy in the future.

\section{Clinical Features}

The most well-defined subgroup of BPS/IC is the bladdercentric Hunner lesion phenotype, which is pathologically distinct from the non-Hunner lesion, systemic phenotype [27••]. Although lesion-targeted therapy can be effective for some patients with Hunner's lesions, there is mixed evidence on whether these phenotypes predict response to other oral or intravesical therapies [28]. Clinical features between these groups also vary, and so focussing on other clinical findings may enable the identification of further phenotypes of BPS/ IC.

\section{Phenotype Based on Characteristics of Pain}

Careful questioning regarding the characteristics, severity and location of pain, and associated urological symptoms, may reveal different underlying subgroups of BPS/IC. Survey responses from 214 women with BPS/IC revealed that pain on vaginal penetration was significantly more common in women without Hunner's lesions than those with Hunner's lesions [29]. Although there was no difference between the groups in terms of pain severity scores, the characteristics of sharp, stabbing and hot burning pain were more likely to identify Hunner's lesions patients, whilst aching, cramping or tender pain was more commonly described by non-Hunner's lesion patients. Similarly, another small study reported more nocturia episodes in patients with Hunner's lesions compared with those without (ICSI nocturia symptom score 4 vs. 3, $p=$ 0.007), but the clinical relevance of this difference is questionable, and there was no difference in bladder hypersensitivity features (e.g. painful bladder filling) between the two groups [30]. However, larger studies have reported greater urinary frequency, nocturia and ICSI symptom scores in patients with Hunner's lesions [31, 32]. Painful bladder filling is a classic feature of BPS/IC with studies reporting the presence of this symptom in $56-76 \%$ of patients [33]. This is distinct from painful urgency, which is a separate symptom reported by 65-87\% of patients with BPS/IC [34, 35]. A recent study of men and women with urological chronic pelvic pain syndrome reported that the prevalence of painful filling or painful urgency, or both, was $88 \%$ in women, and this was associated with more severe urological symptoms as well as more generalised non-urological symptoms; $12 \%$ reported neither characteristic and may represent a separate phenotype of BPS/IC [36]. This would suggest that careful evaluation of symptomatology may enable the identification of discrete patient subgroups, but the evidence is mixed in this regard and requires further study.

\section{Phenotype Based on Co-existing Somatic Conditions}

The presence of systemic, non-urological pain as well as certain associated medical conditions is thought to be representative of a separate phenotype of BPS/IC, and a recent study reported that $75 \%$ of patients with urological chronic pelvic pain syndrome reported pain outside the pelvis $[32,37]$. One study revealed a subgroup of patients who described prodromal symptoms (milder chronic urinary symptoms) for a median of 22 years before the onset of BPS/IC, and these patients were thought to represent a unique phenotype compared with those with no prodromal symptoms [38]. The presence of UTIs in the year prior to BPS/IC onset and co-existence of four or more non-bladder syndromes were seen more commonly in this particular prodromal phenotype [39]. In a study of 639 patients, the prevalence of chronic disease, fibromyalgia, migraines and temperomandibular joint dysfunction was higher in non-Hunner's lesion patients compared with those with Hunner's lesions or asymptomatic controls [32]. A review of eleven studies suggested that in a subgroup of patients, BPS/IC may be one manifestation of a more systemic functional somatic syndrome (FSS) (somatic complaints accompanied by disproportionate thoughts, feelings and behaviours related to such symptoms) [40]. BPS/IC was associated with FSSs and the following conditions were linked to FSSs: fibromyalgia, chronic fatigue syndrome, irritable bowel syndrome, temperomandibular disorder, migraine, depression, anxiety, low back pain, allergies, asthma, sicca syndrome and vulvodynia. Furthermore, studies have shown that in a proportion of patients, multiple FSSs were a risk factor for the development of BPS/IC [41]. Although the exact mechanism of this association is unclear, a recent study of 39 patients with refractory chronic pelvic pain who underwent punch biopsies of the lower extremities revealed the presence of small fibre polyneuropathy in 64\% [42]. In this study, a large proportion of patients suffered from many of the somatic syndromes described above. Identification of this subgroup of BPS/IC is important as patients may be suitable for targeted therapy, and this is a promising area for future research [43].

The presence of multiple sensitivities may represent a distinct phenotype from that associated with other somatic syndromes, and a recent case-control study of 51 patients found an association with multiple sensitivities (at least 3 confirmed allergies/sensitivities to medications and/or environmental factors) in a subgroup of patients with BPS/IC [44].

Recent reports have also suggested a distinct phenotype of BPS/IC associated with autoimmune conditions. A nationwide population-based cohort study of 11,526 patients with primary Sjogrens syndrome in Taiwan revealed an increased risk of BPS/IC in this cohort, with a hazard ratio of 2.34 (95\% 
CI 1.59-3.44, $p<0.01$ ) [45]. A similar study revealed a higher incidence of BPS/IC in patients with systemic lupus erythematosus, with a hazard ratio of 2.45 (95\% CI $1.57-3.27$, $p<0.05$ ) [46]. Further research is required to determine whether phenotypes of BPS/IC associated with autoimmune conditions could benefit from targeted immunomodulatory therapies [47].

\section{Phenotype Based on Psychological Comorbidity}

Psychological comorbidity, such as depression, anxiety, stress and coping, and the psychological effects of trauma have all been shown to accompany and intensify symptoms of BPS/ IC, and are associated with poorer response to treatment [48, 49]. Screening for catastrophising, coping and depression and addressing these psychosocial complaints are recommended in order to improve the efficacy of therapy and overall quality of life [2•, 50]. A recent systematic review of 34 studies of patients with BPS/IC revealed that the average population questionnaire scores were in the range of that seen in clinical depression and generalised anxiety disorder, and that although evidence for treatment is lacking, self-management techniques (guided imagery/self-hypnosis, mindfulness and online health education) may improve symptoms and well-being [51].

Another related phenotype of BPS/IC may be the psychosocial disorders that result from trauma. The relationship between exposure to abuse and BPS/IC is commonly seen in clinical practice. A recent case-control study questioned 94 patients with BPS/IC about traumatising childhood events and psychiatric symptoms [52]. It was found that traumatising events from close others during childhood were associated with the greatest anxiety and dissociative symptoms, which was the factor most likely to distinguish BPS/IC patients from controls. Similarly, the same group reported that the patients who had suffered traumatising childhood events from close others were more likely to have larger bladder capacities (as opposed to that seen in patients with Hunner's lesions) and low affective alexithymia (a key feature of functional somatic syndromes) [53]. In support of this, a recent study of 62 BPS/ IC patients reported that $42 \%$ met the diagnostic criteria for post-traumatic stress disorder, with a significantly higher incidence of lifetime sexual abuse and childhood trauma in this group [54]. Identifying these traumatic events as part of a multidisciplinary specialist team approach is therefore essential in order to optimise management of these conditions. Whether a distinct set of pathogenic factors exists in this subgroup of patients with a history of childhood trauma remains to be studied.

\section{Phenotype Based on Radiological Features}

Recent studies using MRI have increased our understanding of the possible pathophysiological basis of BPS/IC and may improve our ability to identify specific clinical phenotypes. A matched case-control study of 15 women with BPS/IC and 15 controls showed that MRI demonstrated evidence of pelvic floor hypertonicity in those with BPS, based on shortened levator muscles, increased posterior puborectalis angles and decreased puborectal distances [55]. Another study attempted to use diffusion-weighted MRI to diagnose BPS/IC. In this study of 106 patients, a high signal intensity of the bladder wall was related to the presence of BPS/IC (sensitivity $28 \%$, specificity $88 \%$ ), whilst no signal intensity was related to the absence of a BPS/IC (sensitivity 96\%, specificity 29\%) [56]. Using intravesical contrast, it was reported that MRI can also be used to assess bladder permeability, with greater contrast enhancement in BPS/IC compared with controls [57]. The role of MRI in phenotyping patients may be promising but requires further investigation. Another radiological study using colour Doppler ultrasound of the internal iliac arteries noted significantly reduced bladder blood flow in patients with BPS [58]. These preliminary studies require confirmation in larger, well-designed studies comparing Hunner's lesion vs. non-Hunner's lesion patients in order to assess the utility of these radiological findings in aiding phenotyping and subsequent targeted treatments.

Radiological studies of the central nervous system have also identified neuropathological changes that could be used to stratify patients with BPS/IC in the future. Diffusion tensor imaging (DTI) has identified numerous white matter abnormalities in patients with BPS/IC which correlated with symptom severity, and further research is required to determine whether features vary based on the presence of Hunner's lesions and duration of symptoms, and in response to different treatments [59]. A recent meta-analysis reported greater efficacy for sacral neuromodulation treatment in patients with non-Hunner's lesion disease compared with those with Hunner's lesions, and this may be related to a central mechanism of action, but predicting which subgroups will derive the greatest efficacy from treatment remains to be studied [60].

\section{Phenotype Based on Urodynamic Parameters}

Although urodynamics is not recommended by international guidelines as part of the standard diagnostic investigation of BPS/IC [3], certain urodynamic findings may be useful in identifying novel phenotypes. A cross-sectional study compared the urodynamic findings of 24 patients with BPS/IC with those of 28 patients with severe overactive bladder (OAB). Volumes at first sensation, normal desire, strong desire and maximal capacity during filling cystometry were significantly lower in the BPS/IC group, and similar findings were reported in patients with Hunner's lesions compared with those without $[61,62]$. It has been found that patients with BPS/IC and myofascial pelvic pain may also exhibit distinct voiding phenotypes, with lower average and peak 
flow rates, but this requires further study using invasive urodynamics [63]. Several urodynamic features may, therefore, be useful in identifying specific phenotypes of BPS/IC.

\section{Cystoscopic Features}

It is recommended that cystoscopy be performed in patients with BPS/IC to exclude other diseases and to identify the Hunner's lesion phenotype which would benefit from ulcertargeted therapy $[7 \cdot \bullet]$.

\section{Phenotype: Hunner Lesion Disease}

The Hunner lesion phenotype is a well-defined and clearly identified phenotype with distinct clinical and pathological features, and for which targeted therapy with fulguration or triamcinolone injection has been shown to be effective [64]. A recent study has investigated whether the extent of the Hunner's lesion affects symptom severity or response to treatment [65•]. It was found that more extensive lesions were associated with more severe symptoms and a smaller bladder capacity, but did not predict the need for repeat hydrodistension [65•]. However, it is not clear whether extensive Hunner's lesion disease represents a progressive, more severe form of the disease, or whether it is a different phenotype from isolated Hunner's lesions, with different responses to therapy, and this is an interesting area for further research.

\section{Phenotype: Low Bladder Capacity}

The bladder-centric Hunner's lesion phenotype is associated with a low anatomical bladder capacity under anaesthesia - an indication of the destructive inflammatory nature of Hunner's lesion disease which ultimately results in bladder contracture [66•]. Recent studies have shown that low anaesthetic bladder capacity is associated with more severe symptoms, but a lower likelihood of psychosocial complaints or other systemic pain syndromes $[67,68]$. Furthermore, patients with an anaesthetic bladder capacity of $<400 \mathrm{ml}$ are more likely to have Hunner's lesions and have histological evidence of significantly more acute and chronic inflammation compared with patients with a bladder capacity $>400 \mathrm{ml}$ [69].

These studies confirm that low anaesthetic bladder capacity represents a specific, bladder-centric phenotype of BPS/IC, and further investigation into treatments that may reverse this process may enable personalised therapy in the future.

\section{Histopathological Features}

Histopathological analysis of bladder biopsies is a key component of the International Society for the Study of BPS (ESSIC) classification system, and so is recommended by ESSIC guidelines to confirm the diagnosis and for the purpose of phenotyping patients [1••]. Key features of Hunner's lesion disease include ulceration and epithelial denudation, granulation tissue, chronic inflammation including inflammatory cell patterns (lymphoplasmacytic and plasmacytic), fibrosis and elevated mast cell counts, as opposed to the non-specific findings seen with the non-Hunner's phenotype [7••, 70]. However, data regarding mast cells is mixed with a recent study showing higher levels of mast cells in non-Hunner's lesion patients [71]. Interestingly, a recent study has shown that histamine receptor expression is upregulated in patients with BPS/IC, but this did not predict response to antihistamine treatment [72]. The question of whether an elevated mast cell count represents a distinct phenotype of BPS independent of the presence of Hunner's lesions remains to be determined.

Unique cytokine patterns have been identified in patients with Hunner's lesions, with IL-6, 10 and 17A, and iNOS mRNA levels found to be significantly elevated in this group [73]. Whether these findings are specific to a subgroup of patients with Hunner's lesions, or whether they occur in all patients, remains to be studied. Future studies should assess whether the presence of these cytokines identifies a subgroup of patients that may benefit from novel treatments.

Angiogenesis has been reported to distinguish BPS/IC from controls, and increased expression of CD31 was strongly correlated with urinary frequency and bladder pain symptoms [74]. A recent study of 33 patients with BPS/IC reported a distinct gene expression profile in patients with the Hunner's lesion phenotype compared with those without Hunner's lesions and asymptomatic controls, with overexpression of VEGF and BAFF genes, correlating with symptom severity [75]. Identification of these genes may enable phenotyping of BPS/IC patients and targeting of therapy in the future, but this remains to be studied.

\section{Phenotype System: UPOINT}

The UPOINT clinical phenotyping system has proven effective for the clinical management of patients with chronic pelvic pain, focussing on 6 different clinical domains (urological, psychosocial, organ specific, infection, neurologic/systemic and tenderness of the muscles) in order to guide multimodal therapy $[76 \cdot \bullet$. However, this system of clinical phenotyping may be less effective in patients with BPS/IC because the urological and organ-specific aspects are, by definition, present in all. Therefore, a novel clinical phenotyping system which removes these domains and adds a domain for the presence of Hunner's lesions-INPUT (infection, neurologic/systemic, psychosocial, ulcers and tenderness of muscles) - has been suggested [77]. The aim is to ensure that all aspects are evaluated for each patient with BPS/IC so that targeted treatment for all domains can be provided simultaneously, rather than offering a sequential tiered approach. This system was studied in 239 patients and found to replicate the validity and 
clinical utility of UPOINT in chronic pelvic pain syndrome [77]. This system seems to provide a clinically useful, systematic approach to ensure that the most well-studied clinical phenotypes of BPS/IC are investigated and treated, although there are likely to be a large number of as-yet unstudied phenotypes, as described in this review, that will change the way we manage patients with BPS/IC in the future.

\section{Response to Treatment}

Overall treatment efficacy for most BPS/IC treatments is modest and temporary. It is believed that the accurate phenotyping of patients will optimise therapeutic outcomes. However, a small number of comparative studies that assess treatment outcomes based on phenotype have been disappointing, apart from lesion-targeted therapy as described below. A small retrospective study compared the outcomes of amitriptyline treatment in patients with BPS/IC stratified according to ESSIC criteria and found no difference in efficacy between ESSIC type 1 and ESSIC type 3 patients [28]. Similarly, another retrospective study comparing antihistamine responders to non-responders found no correlation between the expression levels of histamine receptors and treatment outcome, although patients were not stratified by cystoscopic features [72]. Outcomes from intravesical hyaluronic acid instillations cannot be predicted by the presence or absence of Hunner's lesions, and data for superior efficacy for intravesical dimethylsulfoxide (DMSO) in Hunner's lesion patients compared with non-Hunner's lesion patients comes from a small, retrospective study $[78,79]$. Although evidence for efficacy of intravesical injection of botulinum toxin A in BPS/IC comes from randomised trials [80], there has been no reported difference in efficacy rates when comparing Hunner's with nonHunner's lesion patients in non-randomised studies [81, 82]. Recent systematic reviews and meta-analyses have suggested success rates for sacral neuromodulation in this group of 60 $98 \%$, but patients with chronic pelvic pain were likely to have a better response than those with BPS/IC specifically [60, 83]. However, there has not been any satisfactory comparative data between different phenotypes of BPS/IC and these systematic reviews are limited by the heterogenous and low-quality evidence base.

Despite this data, the utility of accurate phenotyping has been demonstrated by lesion-targeted therapy for Hunner's lesion disease. Transurethral fulguration (with diathermy or laser), intra-lesional triamcinolone injection and transurethral resection of Hunner's lesions have all demonstrated efficacy in this difficult-to-treat group [7••]. Furthermore, although the literature is mixed and studies do not always report the results of different phenotypes of BPS separately, it is thought that those with Hunner's lesion disease and small anaesthetic bladder capacity are more likely to benefit from major surgical reconstruction $[84,85]$.
Overall, although accurate phenotyping is essential for optimisation of therapy, the current evidence base is limited by considerable heterogeneity in definitions of BPS/ IC, lack of consistent phenotyping and consistent outcome reporting and a paucity of well-powered randomised trials. Future studies should accurately stratify patients by phenotype and report results separately for these different groups, and should be randomised and adequately powered. In this regard, the Multidisciplinary Approach to the Study of Chronic Pelvic Pain (MAPP) Research Network is a unique and revolutionary study group that is providing high-quality evidence regarding the investigation and management of BPS/IC [86••].

\section{Conclusion}

The aim of phenotyping patients with BPS/IC is to improve our understanding of the pathophysiological mechanisms of disease and to allow personalised, tailored treatment in order to optimise outcomes. Two clear phenotypes have been extensively reported in the literatureHunner's lesion disease and non-Hunner's lesion disease. Hunner's lesion disease is a bladder-centric condition with distinct clinical, cystoscopic and pathological features, whereas non-Hunner's disease is a broader, systemic condition associated with other somatic and psychosocial syndromes. However, many other phenotypes are likely to exist based on biochemical, pathological or clinical features, as summarised in this review. Although promising, these features require further study in order to assess their role in phenotyping patients and in directing successful treatment strategies.

\section{Compliance with Ethical Standards}

Conflict of Interest Dr. Malde and Dr. Sahai report an educational grant from Medtronic Ltd. and an unrestricted educational grant from Boston Scientific. Dr. Sahai reports speaker fees from Ferring Pharmaceuticals and an unrestricted educational grant from Allergan.

Human and Animal Rights and Informed Consent This article does not contain any studies with human or animal subjects performed by any of the authors.

Funding This paper was made Open Access with financial support from King's College London.

Open Access This article is licensed under a Creative Commons Attribution 4.0 International License, which permits use, sharing, adaptation, distribution and reproduction in any medium or format, as long as you give appropriate credit to the original author(s) and the source, provide a link to the Creative Commons licence, and indicate if changes were made. The images or other third party material in this article are included in the article's Creative Commons licence, unless indicated otherwise in a credit line to the material. If material is not included in the article's 
Creative Commons licence and your intended use is not permitted by statutory regulation or exceeds the permitted use, you will need to obtain permission directly from the copyright holder. To view a copy of this licence, visit http://creativecommons.org/licenses/by/4.0/.

\section{References}

Papers of particular interest, published recently, have been

highlighted as:

- Of importance

-• Of major importance

1.• van de Merwe JP, Nordling J, Bouchelouche P, Bouchelouche K, Cervigni M, Daha LK, et al. Diagnostic criteria, classification, and nomenclature for painful bladder syndrome/interstitial cystitis: an ESSIC proposal. Eur Urol. 2008;53(1):60-7. https://doi.org/10. 1016/j.eururo.2007.09.019 The ESSIC classification system for BPS/IC.

2. Engeler D BA, Borovicka J, Cottrell AM, Dinis-Oliveira P, Elneil $\mathrm{S}$, Hughes J, Messelink EJ, de C Williams AC. EUA guidelines on chronic pelvic pain. Retrieved from http://uroweborg/guideline/ chronic-pelvic-pain/ accessed May 2020. EAU guidelines on management of chronic pelvic pain.

3. Malde S, Palmisani S, Al-Kaisy A, Sahai A. Guideline of guidelines: bladder pain syndrome. BJU Int. 2018;122(5):729-43. https://doi.org/10.1111/bju.14399.

4. Baranowski AP, Abrams P, Berger RE, Buffington CA, de CWAC, Hanno P, et al. Urogenital pain-time to accept a new approach to phenotyping and, as a consequence, management. Eur Urol. 2008;53(1):33-6. https://doi.org/10.1016/j.eururo.2007.10.010.

5. Hunner GL. A rare type of bladder ulcer in women; report of cases. Boston Med Surg J. 1915;172(18):660-4. https://doi.org/10.1056/ NEJM191505061721802.

6. Malde S, Apostilidis A, Selai C, Rahnama'i MS, Marcelissen T, Cardozo L, et al. Botulinum toxin A for refractory OAB and idiopathic urinary retention: can phenotyping improve outcome for patients: ICI-RS 2019? Neurourol Urodyn. 2019. https://doi.org/10. 1002/nau.24207.

7.• Fall M, Nordling J, Cervigni M, Dinis Oliveira P, Fariello J, Hanno $\mathrm{P}$ et al. Hunner lesion disease differs in diagnosis, treatment and outcome from bladder pain syndrome: an ESSIC working group report. Scand J Urol. 2020:1-8. https://doi.org/10.1080/21681805. 2020.1730948 Excellent recent review focussing on Hunner's lesion disease.

8. Magalhaes TF, Baracat EC, Doumouchtsis SK, Haddad JM. Biomarkers in the diagnosis and symptom assessment of patients with bladder pain syndrome: a systematic review. Int Urogynecol J. 2019;30(11):1785-94. https://doi.org/10.1007/s00192-019-040759.

9. Keay SK, Zhang CO, Shoenfelt J, Erickson DR, Whitmore K, Warren JW, et al. Sensitivity and specificity of antiproliferative factor, heparin-binding epidermal growth factor-like growth factor, and epidermal growth factor as urine markers for interstitial cystitis. Urology. 2001;57(6 Suppl 1):9-14. https://doi.org/10.1016/s00904295(01)01127-x.

10. Zhang CO, Li ZL, Kong CZ. APF, HB-EGF, and EGF biomarkers in patients with ulcerative vs. non-ulcerative interstitial cystitis. BMC urology. 2005;5:7. https://doi.org/10.1186/1471-2490-5-7.

11. Erickson DR, Kunselman AR, Bentley CM, Peters KM, Rovner ES, Demers LM, et al. Changes in urine markers and symptoms after bladder distention for interstitial cystitis. J Urol. 2007;177(2): 556-60. https://doi.org/10.1016/j.juro.2006.09.029.
12. Vera PL, Preston DM, Moldwin RM, Erickson DR, Mowlazadeh $\mathrm{B}$, Ma F, et al. Elevated urine levels of macrophage migration inhibitory factor in inflammatory bladder conditions: a potential biomarker for a subgroup of interstitial cystitis/bladder pain syndrome patients. Urology. 2018;116:55-62. https://doi.org/10.1016/ j.urology.2018.02.039.

13. Niimi A, Igawa $Y$, Aizawa N, Honma T, Nomiya A, Akiyama Y, et al. Diagnostic value of urinary CXCL10 as a biomarker for predicting Hunner type interstitial cystitis. Neurourol Urodyn. 2018;37(3):1113-9. https://doi.org/10.1002/nau.23431.

14. Parker KS, Crowley JR, Stephens-Shields AJ, van Bokhoven A, Lucia MS, Lai HH, et al. Urinary metabolomics identifies a molecular correlate of interstitial cystitis/bladder pain syndrome in a Multidisciplinary Approach to the Study of Chronic Pelvic Pain (MAPP) Research Network cohort. EBioMedicine. 2016;7:16774. https://doi.org/10.1016/j.ebiom.2016.03.040.

15. Schrepf A, Bradley CS, O'Donnell M, Luo Y, Harte SE, Kreder K, et al. Toll-like receptor 4 and comorbid pain in interstitial cystitis/ bladder pain syndrome: a multidisciplinary approach to the study of chronic pelvic pain research network study. Brain Behav Immun. 2015;49:66-74. https://doi.org/10.1016/j.bbi.2015.03.003.

16. Offiah I, Didangelos A, Dawes J, Cartwright R, Khullar V, Bradbury EJ, et al. The expression of inflammatory mediators in bladder pain syndrome. Eur Urol. 2016;70(2):283-90. https://doi. org/10.1016/j.eururo.2016.02.058.

17. Choi D, Han JY, Shin JH, Ryu CM, Yu HY, Kim A, et al. Downregulation of WNT11 is associated with bladder tissue fibrosis in patients with interstitial cystitis/bladder pain syndrome without Hunner lesion. Sci Rep. 2018;8(1):9782. https://doi.org/10. 1038/s41598-018-28093-7.

18. Swamy S, Kupelian AS, Khasriya R, Dharmasena D, Toteva H, Dehpour T, et al. Cross-over data supporting long-term antibiotic treatment in patients with painful lower urinary tract symptoms, pyuria and negative urinalysis. Int Urogynecol J. 2019;30(3):40914. https://doi.org/10.1007/s00192-018-3846-5.

19. Khasriya R, Khan S, Lunawat R, Bishara S, Bignall J, Malone-Lee $\mathrm{M}$, et al. The inadequacy of urinary dipstick and microscopy as surrogate markers of urinary tract infection in urological outpatients with lower urinary tract symptoms without acute frequency and dysuria. J Urol. 2010;183(5):1843-7. https://doi.org/10.1016/j. juro.2010.01.008.

20. Aydogan TB, Gurpinar O, Eser OK, Mathyk BA, Ergen A. A new look at the etiology of interstitial cystitis/bladder pain syndrome: extraordinary cultivations. Int Urol Nephrol. 2019;51(11):1961-7. https://doi.org/10.1007/s11255-019-02248-5.

21. Zhang QH, Shen XC, Zhou ZS, Chen ZW, Lu GS, Song B. Decreased nanobacteria levels and symptoms of nanobacteriaassociated interstitial cystitis/painful bladder syndrome after tetracycline treatment. Int Urogynecol J. 2010;21(1):103-9. https://doi. org/10.1007/s00192-009-0994-7.

22. Siddiqui H, Lagesen K, Nederbragt AJ, Jeansson SL, Jakobsen KS. Alterations of microbiota in urine from women with interstitial cystitis. BMC Microbiol. 2012;12:205. https://doi.org/10.1186/14712180-12-205.

23. Nickel JC, Stephens A, Landis JR, Mullins C, van Bokhoven A, Anger JT, et al. Urinary fungi associated with urinary symptom severity among women with interstitial cystitis/bladder pain syndrome (IC/BPS). World J Urol. 2020;38(2):433-46. https://doi.org/ 10.1007/s00345-019-02764-0.

24. Eisen DP, Fraser IR, Sung LM, Finlay M, Bowden S, O'Connell H. Decreased viral load and symptoms of polyomavirus-associated chronic interstitial cystitis after intravesical cidofovir treatment. Clin Infect Dis. 2009;48(9):e86-8. https://doi.org/10.1086/597827.

25. Jhang JF, Hsu YH, Peng CW, Jiang YH, Ho HC, Kuo HC. EpsteinBarr virus as a potential etiology of persistent bladder inflammation 
in human interstitial cystitis/bladder pain syndrome. J Urol. 2018;200(3):590-6. https://doi.org/10.1016/j.juro.2018.03.133.

26. Van der Aa F, Beckley I, de Ridder D. Polyomavirus BK-a potential new therapeutic target for painful bladder syndrome/interstitial cystitis? Med Hypotheses. 2014;83(3):317-20. https://doi.org/10. 1016/j.mehy.2014.06.004

27.• Akiyama Y, Hanno P. Phenotyping of interstitial cystitis/bladder pain syndrome. Int J Urol. 2019;26 Suppl 1:17-19. doi:https://doi. org/10.1111/iju.13969. Summary of the International Consultation on Interstitial Cystitis Japan workshop on phenotyping in BPS/IC.

28. Sun Y, Fang Z, Ding Q, Zheng J. Effect of amitriptyline in treatment interstitial cystitis or bladder pain syndrome according to two criteria: does ESSIC criteria change the response rate? Neurourol Urodyn. 2014;33(3):341-4. https://doi.org/10.1002/nau.22407.

29. Killinger KA, Boura JA, Peters KM. Pain in interstitial cystitis/ bladder pain syndrome: do characteristics differ in ulcerative and non-ulcerative subtypes? Int Urogynecol J. 2013;24(8):1295-301. https://doi.org/10.1007/s00192-012-2003-9.

30. Van Moh F, Vetter J, Lai HH. Comparison of urologic and nonurologic presentation in interstitial cystitis/bladder pain syndrome patients with and without Hunner lesions. Neurourol Urodyn. 2018;37(8):2911-8. https://doi.org/10.1002/nau.23812.

31. Doiron RC, Tolls V, Irvine-Bird K, Kelly KL, Nickel JC. Clinical Phenotyping does not differentiate Hunner lesion subtype of interstitial cystitis/bladder pain syndrome: a relook at the role of cystoscopy. J Urol. 2016;196(4):1136-40. https://doi.org/10.1016/j.juro. 2016.04.067.

32. Peters KM, Killinger KA, Mounayer MH, Boura JA. Are ulcerative and nonulcerative interstitial cystitis/painful bladder syndrome 2 distinct diseases? A Study of Coexisting Conditions Urology. 2011;78(2):301-8. https://doi.org/10.1016/j.urology.2011.04.030.

33. Warren JW, Brown J, Tracy JK, Langenberg P, Wesselmann U, Greenberg P. Evidence-based criteria for pain of interstitial cystitis/ painful bladder syndrome in women. Urology. 2008;71(3):444-8. https://doi.org/10.1016/j.urology.2007.10.062.

34. Clemens JQ, Bogart LM, Liu K, Pham C, Suttorp M, Berry SH. Perceptions of "urgency" in women with interstitial cystitis/bladder pain syndrome or overactive bladder. Neurourol Urodyn. 2011;30(3):402-5. https://doi.org/10.1002/nau.20974.

35. Greenberg P, Brown J, Yates T, Brown V, Langenberg P, Warren JW. Voiding urges perceived by patients with interstitial cystitis/ painful bladder syndrome. Neurourol Urodyn. 2008;27(4):287-90. https://doi.org/10.1002/nau.20516.

36. Lai HH, Krieger JN, Pontari MA, Buchwald D, Hou X, Landis JR. Painful bladder filling and painful urgency are distinct characteristics in men and women with urological chronic pelvic pain syndromes: a MAPP Research Network study. J Urol. 2015;194(6): 1634-41. https://doi.org/10.1016/j.juro.2015.05.105.

37. Lai HH, Jemielita T, Sutcliffe S, Bradley CS, Naliboff B, Williams DA, et al. Characterization of whole body pain in urological chronic pelvic pain syndrome at baseline: a MAPP Research Network study. J Urol. 2017;198(3):622-31. https://doi.org/10.1016/j.juro. 2017.03.132

38. Warren JW, Wesselmann U, Greenberg P, Clauw DJ. Urinary symptoms as a prodrome of bladder pain syndrome/interstitial cystitis. Urology. 2014;83(5):1035-40. https://doi.org/10.1016/j. urology.2014.01.012.

39. Warren JW, Jian N, Gallicchio L, Wu D, Clauw DJ. Prodrome and non-prodrome phenotypes of bladder pain syndrome/interstitial cystitis. Urology. 2018;118:52-8. https://doi.org/10.1016/j. urology.2018.05.004.

40. Warren JW. Bladder pain syndrome/interstitial cystitis as a functional somatic syndrome. J Psychosom Res. 2014;77(6):510-5. https://doi.org/10.1016/j.jpsychores.2014.10.003.
41. Warren JW, Wesselmann U, Morozov V, Langenberg PW. Numbers and types of nonbladder syndromes as risk factors for interstitial cystitis/painful bladder syndrome. Urology. 2011;77(2):313-9. https://doi.org/10.1016/j.urology.2010.08.059.

42. Chen A, De E, Argoff C. Small fiber polyneuropathy is prevalent in patients experiencing complex chronic pelvic pain. Pain Medicine (Malden, Mass). 2019;20(3):521-7. https://doi.org/10.1093/pm/ pny001.

43. Matthews CA, Deveshwar SP, Evans RJ, Badlani G, Walker SJ. Small fiber polyneuropathy as a potential therapeutic target in interstitial cystitis/bladder pain syndrome. Int Urogynecol J. 2019;30(11):1817-20. https://doi.org/10.1007/s00192-019-04011$\mathrm{x}$.

44. Fuoco MB, Irvine-Bird K, Curtis NJ. Multiple sensitivity phenotype in interstitial cystitis/bladder pain syndrome. Can Urol Assoc J $=$ Journal de l'Association des urologues du Canada. 2014;8(1112):E758-61. https://doi.org/10.5489/cuaj.2031.

45. Lee CK, Tsai CP, Liao TL, Huang WN, Chen YH, Lin CH, et al. Overactive bladder and bladder pain syndrome/interstitial cystitis in primary Sjogren's syndrome patients: a nationwide populationbased study. PLoS One. 2019;14(11):e0225455. https://doi.org/ 10.1371/journal.pone.0225455.

46. Wen JY, Lo TS, Chuang YC, Ho CH, Long CY, Law KS, et al. Risks of interstitial cystitis among patients with systemic lupus erythematosus: a population-based cohort study. Int J Urol. 2019;26(9):897-902. https://doi.org/10.1111/iju.14065.

47. Bosch PC. A randomized, double-blind, placebo-controlled trial of certolizumab pegol in women with refractory interstitial cystitis/ bladder pain syndrome. Eur Urol. 2018;74(5):623-30. https://doi. org/10.1016/j.eururo.2018.07.026.

48. Naliboff BD, Stephens AJ, Afari N, Lai H, Krieger JN, Hong B, et al. Widespread psychosocial difficulties in men and women with urologic chronic pelvic pain syndromes: case-control findings from the Multidisciplinary Approach to the Study of Chronic Pelvic Pain Research Network. Urology. 2015;85(6):1319-27. https://doi.org/ 10.1016/j.urology.2015.02.047.

49. Koh JS, Ko HJ, Wang SM, Cho KJ, Kim JC, Lee SJ, et al. The impact of depression and somatic symptoms on treatment outcomes in patients with chronic prostatitis/chronic pelvic pain syndrome: a preliminary study in a naturalistic treatment setting. Int J Clin Pract. 2014;68(4):478-85. https://doi.org/10.1111/ijcp.12340.

50. Muere A, Tripp DA, Nickel JC, Kelly K-L, Mayer R, Pontari M, et al. Depression and coping behaviors are key factors in understanding pain in interstitial cystitis/bladder pain syndrome. Pain Manag Nurs. 2018;19(5):497-505. https://doi.org/10.1016/j.pmn. 2017.11.001.

51. McKernan LC, Walsh CG, Reynolds WS, Crofford LJ, Dmochowski RR, Williams DA. Psychosocial co-morbidities in interstitial cystitis/bladder pain syndrome (IC/BPS): a systematic review. Neurourol Urodyn. 2018;37(3):926-41. https://doi.org/10. 1002/nau.23421.

52. Chiu CD, Lee MH, Chen WC, Ho HL, Wu HC. Childhood trauma perpetrated by close others, psychiatric dysfunction, and urological symptoms in patients with interstitial cystitis/bladder pain syndrome. J Psychosom Res. 2017;93:90-5. https://doi.org/10.1016/j. jpsychores.2016.12.014.

53. Chiu CD, Lee MH, Chen WC, Ho HL, Wu HC. Alexithymia and anesthetic bladder capacity in interstitial cystitis/bladder pain syndrome. J Psychosom Res. 2017;100:15-21. https://doi.org/10. 1016/j.jpsychores.2017.06.019

54. McKernan LC, Johnson BN, Reynolds WS, Williams DA, Cheavens JS, Dmochowski RR, et al. Posttraumatic stress disorder in interstitial cystitis/bladder pain syndrome: relationship to patient phenotype and clinical practice implications. Neurourol Urodyn. 2019;38(1):353-62. https://doi.org/10.1002/nau.23861. 
55. Ackerman AL, Lee UJ, Jellison FC, Tan N, Patel M, Raman SS, et al. MRI suggests increased tonicity of the levator ani in women with interstitial cystitis/bladder pain syndrome. Int Urogynecol J. 2016;27(1):77-83. https://doi.org/10.1007/s00192-015-2794-6.

56. Charlanes A, Boudghene F, Chesnel C, Ciofu C, Le Breton F, Jousse $\mathrm{M}$, et al. Diffusion-weighted magnetic resonance imaging: a new tool for the diagnosis of bladder pain syndrome/interstitial cystitis. Urol Int. 2019;102(1):109-12. https://doi.org/10.1159/ 000493507.

57. Towner RA, Wisniewski AB, Wu DH, Van Gordon SB, Smith N, North JC, et al. A feasibility study to determine whether clinical contrast enhanced magnetic resonance imaging can detect increased bladder permeability in patients with interstitial cystitis. J Urol. 2016;195(3):631-8. https://doi.org/10.1016/j.juro.2015.08.077.

58. Ozcaglayan O, Akgul M, Yazici C, Ozcaglayan TI, Malak A, Dogru MM. Is bladder blood flow an etiologic factor for the bladder pain syndrome? Neurourol Urodyn. 2019;38(4):1135-41. https:// doi.org/10.1002/nau.23969.

59. Farmer MA, Huang L, Martucci K, Yang CC, Maravilla KR, Harris $\mathrm{RE}$, et al. Brain white matter abnormalities in female interstitial cystitis/bladder pain syndrome: a MAPP network neuroimaging study. J Urol. 2015;194(1):118-26. https://doi.org/10.1016/j.juro. 2015.02.082

60. Mahran A, Baaklini G, Hassani D, Abolella HA, Safwat AS, Neudecker M, et al. Sacral neuromodulation treating chronic pelvic pain: a meta-analysis and systematic review of the literature. Int Urogynecol J. 2019;30(7):1023-35. https://doi.org/10.1007/ s00192-019-03898-w.

61. Shim JS, Kang SG, Park JY, Bae JH, Kang SH, Park HS, et al. Differences in urodynamic parameters between women with interstitial cystitis and/or bladder pain syndrome and severe overactive bladder. Urology. 2016;94:64-9. https://doi.org/10.1016/j.urology. 2016.04.018.

62. Ahn ST, Jeong HG, Park TY, Kim JW, Park HS, Moon DG, et al. Differences in urodynamic parameters according to the presence of a Hunner lesion in women with interstitial cystitis/bladder pain syndrome. Int Neurourol J. 2018;22(Suppl 1):S55-61. https://doi. org/10.5213/inj.1835044.522.

63. Petrikovets A, Veizi IE, Hijaz A, Mahajan ST, Daneshgari F, Buffington CAT, et al. Comparison of voiding dysfunction phenotypes in women with interstitial cystitis/bladder pain and myofascial pelvic pain: results from the ICEPAC trial. Urology. 2019;126:54-8. https://doi.org/10.1016/j.urology.2019.01.015.

64. Crescenze IM, Gupta P, Adams G, Oldendorf A, Stoffel JT, Romo PGB, et al. Advanced management of patients with ulcerative interstitial cystitis/bladder pain syndrome. Urology. 2019;133:78-83. https://doi.org/10.1016/j.urology.2019.07.036.

65. Akiyama Y, Niimi A, Nomiya A, Yamada Y, Nakagawa T, Fujimura T, et al. Extent of Hunner lesions: the relationships with symptom severity and clinical parameters in Hunner type interstitial cystitis patients. Neurourol Urodyn. 2018;37(4):1441-7. https://doi.org/10.1002/nau.23467 Novel study assessing whether extent of Hunner's lesion impacts symptom severity and response to hydrodistension.

66. Logadottir Y, Fall M, Kabjorn-Gustafsson C, Peeker R. Clinical characteristics differ considerably between phenotypes of bladder pain syndrome/interstitial cystitis. Scand J Urol Nephrol. 2012;46(5):365-70. https://doi.org/10.3109/00365599.2012. 689008 Large study describing clinical differences between phenotypes of BPS/IC.

67. Walker SJ, Zambon J, Andersson KE, Langefeld CD, Matthews CA, Badlani G, et al. Bladder capacity is a biomarker for a bladder centric versus systemic manifestation in interstitial cystitis/bladder pain syndrome. J Urol. 2017;198(2):369-75. https://doi.org/10. 1016/j.juro.2017.02.022.
68. Mazeaud C, Rigaud J, Levesque A, Madec FX, Le Clerc QC, Wack $\mathrm{M}$, et al. Stratification of patients with interstitial cystitis/bladder pain syndrome according to the anatomical bladder capacity. Urology. 2019;123:87-92. https://doi.org/10.1016/j.urology.2018. 07.046 .

69. Schachar JS, Evans RJ, Parks GE, Zambon J, Badlani G, Walker SJ. Histological evidence supports low anesthetic bladder capacity as a marker of a bladder-centric disease subtype in interstitial cystitis/bladder pain syndrome. Int Urogynecol J. 2019;30(11): 1863-70. https://doi.org/10.1007/s00192-019-04038-0.

70. Maeda D, Akiyama Y, Morikawa T, Kunita A, Ota Y, Katoh H, et al. Hunner-type (classic) interstitial cystitis: a distinct inflammatory disorder characterized by pancystitis, with frequent expansion of clonal B-cells and epithelial denudation. PLoS One. 2015;10(11):e0143316. https://doi.org/10.1371/journal.pone. 0143316.

71. Kim A, Han JY, Ryu CM, Yu HY, Lee S, Kim Y, et al. Histopathological characteristics of interstitial cystitis/bladder pain syndrome without Hunner lesion. Histopathology. 2017;71(3): 415-24. https://doi.org/10.1111/his.13235.

72. Shan H, Zhang EW, Zhang P, Zhang XD, Zhang N, Du P, et al. Differential expression of histamine receptors in the bladder wall tissues of patients with bladder pain syndrome/interstitial cystitis significance in the responsiveness to antihistamine treatment and disease symptoms. BMC Urol. 2019;19(1):115. https://doi.org/10. 1186/s12894-019-0548-3.

73. Logadottir Y, Delbro D, Fall M, Gjertsson I, Jirholt P, Lindholm C, et al. Cytokine expression in patients with bladder pain syndrome/ interstitial cystitis ESSIC type 3C. J Urol. 2014;192(5):1564-8. https://doi.org/10.1016/j.juro.2014.04.099.

74. Furuta A, Suzuki Y, Igarashi T, Koike Y, Kimura T, Egawa S, et al. Angiogenesis in bladder tissues is strongly correlated with urinary frequency and bladder pain in patients with interstitial cystitis/ bladder pain syndrome. Int J Urol. 2019;26(Suppl 1):35-40. https://doi.org/10.1111/iju.13972.

75. Akiyama $Y$, Maeda D, Katoh H, Morikawa T, Niimi A, Nomiya A, et al. Molecular taxonomy of interstitial cystitis/bladder pain syndrome based on whole transcriptome profiling by next-generation RNA sequencing of bladder mucosal biopsies. J Urol. 2019;202(2): 290-300. https://doi.org/10.1097/ju.0000000000000234.

76.• Shoskes DA, Nickel JC, Rackley RR, Pontari MA. Clinical phenotyping in chronic prostatitis/chronic pelvic pain syndrome and interstitial cystitis: a management strategy for urologic chronic pelvic pain syndromes. Prostate Cancer Prostatic Dis. 2009;12(2):177-83. https://doi.org/10.1038/pcan.2008.42 Description of the UPOINT classification system for phenotyping patients with chronic pelvic pain.

77. Crane A, Lloyd J, Shoskes DA. Improving the utility of clinical phenotyping in interstitial cystitis/painful bladder syndrome: from UPOINT to INPUT. Can J Urol. 2018;25(2):9250-4.

78. Kim A, Lim B, Song M, Choo MS. Pretreatment features to influence effectiveness of intravesical hyaluronic acid instillation in refractory interstitial cystitis/painful bladder syndrome. Int Neurourol J. 2014;18(3):163-7. https://doi.org/10.5213/inj.2014.18.3.163.

79. Tomoe H. In what type of interstitial cystitis/bladder pain syndrome is DMSO intravesical instillation therapy effective? Transl Androl Urol. 2015;4(6):600-4. https://doi.org/10.3978/j.issn.2223-4683. 2015.09.01.

80. Akiyama Y, Nomiya A, Niimi A, Yamada Y, Fujimura T, Nakagawa $\mathrm{T}$, et al. Botulinum toxin type A injection for refractory interstitial cystitis: a randomized comparative study and predictors of treatment response. Int J Urol. 2015;22(9):835-41. https://doi. org/10.1111/iju.12833.

81. Lee C-L, Kuo H-C. Intravesical botulinum toxin A injections do not benefit patients with ulcer type interstitial cystitis. Pain Physician. 2013;16(2):109-16. 
82. Pinto R, Lopes T, Costa D, Barros S, Silva J, Silva C, et al. Ulcerative and nonulcerative forms of bladder pain syndrome/ interstitial cystitis do not differ in symptom intensity or response to onabotulinum toxin A. Urology. 2014;83(5):1030-4. https://doi. org/10.1016/j.urology.2014.01.018.

83. Wang J, Chen Y, Chen J, Zhang G, Wu P. Sacral neuromodulation for refractory bladder pain syndrome/interstitial cystitis: a global systematic review and meta-analysis. Sci Rep. 2017;7(1):11031. https://doi.org/10.1038/s41598-017-11062-x.

84. Rössberger J, Fall M, Jonsson O, Peeker R. Long-term results of reconstructive surgery in patients with bladder pain syndrome/ interstitial cystitis: subtyping is imperative. Urology. 2007;70(4): 638-42. https://doi.org/10.1016/j.urology.2007.05.028.
85. Osman NI, Bratt DG, Downey AP, Esperto F, Inman RD, Chapple CR. A systematic review of surgical interventions for the treatment of bladder pain syndrome/interstitial cystitis. Eur Urol Focus. 2020. https://doi.org/10.1016/j.euf.2020.02.014.

86.• Clemens JQ, Mullins C, Ackerman AL, Bavendam T, van Bokhoven A, Ellingson BM, et al. Urologic chronic pelvic pain syndrome: insights from the MAPP Research Network. Nat Rev Urol. 2019;16(3):187-200. https://doi.org/10.1038/s41585-0180135-5 Excellent review of the latest evidence produced by the MAPP network.

Publisher's Note Springer Nature remains neutral with regard to jurisdictional claims in published maps and institutional affiliations. 